\title{
Interpapillary muscle distance independently affects severity of functional mitral regurgitation in patients with systolic left ventricular dysfunction
}

Kitae Kim, MD, Shuichiro Kaji, MD, Yoshimori An, MD, Tomohiro Nishino, MD, Tomoko Tani, MD, Takeshi Kitai, MD, and Yutaka Furukawa, MD

Objectives: Surgical papillary muscle (PM) imbrication has been reported to be effective to relieve leaflet tethering in patients with functional mitral regurgitation (FMR). However, the mechanism that contributes to improvement of FMR by shortening the interpapillary muscle distance (IPMD) has not been well investigated. The purpose of the present study was to investigate whether IPMD can affect MR severity independently of PM tethering distance in patients with left ventricular dysfunction (LVD) using multislice computed tomography.

Methods: We analyzed volumetric multislice computed tomography images of mitral apparatus in 83 patients with LVD (ejection fraction $<50 \%$ ): 37 patients with FMR and 46 patients without FMR. By using the original software, we assessed the 3-dimensional geometry of mitral apparatus including IPMD, tethering distances, and mitral tenting volume at end-systole. The severity of FMR was assessed using vena contracta (VC) width by 2-dimensional echocardiography.

Results: Posterior and anterior tethering distance and IPMD were increased significantly in patients with FMR than in those without FMR. Patients with IPMD in the highest tertile had a significantly higher degrees of MR (mean \pm standard error VC width, $4.5 \pm 0.3 \mathrm{~mm}$ ) compared with patients in the lowest and the middle tertiles, adjusting for PM tethering distance (mean \pm standard error VC width, $3.0 \pm 0.4$ and $2.9 \pm 0.3 \mathrm{~mm} ; P<.001$, respectively). Multivariate analysis showed that anteroposterior annular diameter and IPMD were the strongest determinants of FMR severity and mitral tenting volume.

Conclusions: IPMD, which affects leaflet tethering independently of PM tethering distance, was the major determinant of mitral tenting volume and FMR severity in patients with LVD. (J Thorac Cardiovasc Surg 2014; 148:434-40)

Supplemental material is available online.

Functional mitral regurgitation (FMR) is a common complication in patients with ischemic heart disease, which occurs as a consequence of regional or global left ventricular dysfunction (LVD). ${ }^{1}$ Although the exact mechanism causing FMR is still elusive, leaflet tethering by the displaced papillary muscles (PMs) has been shown as an essential pathway of FMR. ${ }^{2,3}$ Accordingly, the distance between posterior PM tips and anterior annulus

From the Department of Cardiovascular Medicine, Kobe City Medical Center General Hospital, Kobe, Japan.

Disclosures: Authors have nothing to disclose with regard to commercial support.

Received for publication Feb 4, 2013; revisions received July 28, 2013; accepted for publication Sept 10, 2013; available ahead of print Nov 4, 2013.

Address for reprints: Shuichiro Kaji, MD, Department of Cardiovascular Medicine, Kobe City Medical Center General Hospital, 2-1-1, Minatojima-Minamimachi, Chuo-ku, Kobe, Japan, 650-0047 (E-mail: skaji@theia.ocn.ne.jp).

0022-5223/\$36.00

Copyright (C) 2014 by The American Association for Thoracic Surgery

http://dx.doi.org/10.1016/j.jtcvs.2013.09.029 has been reported to be a major determinant of FMR severity. ${ }^{4}$ On the other hand, previous clinical studies have shown that reducing the distance between anterior and posterior PMs (interpapillary muscle distance [IPMD]) with the PM imbrication technique was effective to relieve leaflet tethering and correct subvalvular geometry. ${ }^{5-8}$ However, because this technique inevitably corrects the posterior PM location with shortening of PM tethering distance to a certain extent, it is unclear whether shortening the IPMD has an additive effect on reducing FMR beyond correcting the posterior PM tethering distance.

Mediolateral displacement of anterior and the posterior PMs potentially can lift up the central portion of the anterior and posterior leaflets and increase the tethering of whole leaflets with increasing IPMD (Figure 1, middle panel). In contrast, apical displacement of both PMs without increasing the IPMD does not lift up the central portion of both leaflets and result in less leaflet tethering (Figure 1, right panel). Therefore, we hypothesized that the IPMD may affect leaflet tethering independently of the effect of the increased PM tethering distance. 

Abbreviations and Acronyms
ANCOVA $=$ analysis of covariance
$\mathrm{AP}=$ anteroposterior
DCM = dilated cardiomyopathy
FMR = functional mitral regurgitation
IPMD = interpapillary muscle distance
LVD = left ventricular dysfunction
MSCT = multislice computed tomography
$\mathrm{PM} \quad=$ papillary muscle
SE $=$ standard error
3D $=3$-dimensional
2D $=2$-dimensional

standard MSCT coronary angiography. The electrocardiogram was recorded simultaneously to allow retrospective gating and reconstruction of the data at desired phases of the cardiac cycle. To study 3D geometry of the mitral apparatus, the end-systolic data set was reconstructed with a slice thickness of $0.625 \mathrm{~mm}$ and a reconstruction interval of $0.35 \mathrm{~mm}$ at $30 \%$ to $40 \%$ of the RR ( $\mathrm{R}$ wave to $\mathrm{R}$ wave) interval. No additional radiation exposure or contrast agent with specific imaging protocol was necessary for mitral valve analysis.

\section{Echocardiography}

LV end-diastolic volume, end-systolic volume, and ejection fraction were measured as previously described with 2-dimensional (2D) transthoracic echocardiography. ${ }^{14}$ MR severity was assessed semiquantitatively according to the recommendation of the American Society of Echocardiography $^{15}$ as none, mild, moderate, or severe. In addition, MR was quantified by the vena contracta width or the narrowest jet origin in a parasternal or apical long-axis view perpendicular to the coaptation line. ${ }^{16,17}$

Recent advances in 3-dimensional (3D) imaging techniques such as echocardiography, ${ }^{9}$ magnetic resonance imaging, ${ }^{10,11}$ and multislice computed tomography $(\mathrm{MSCT})^{12}$ have provided a better understanding of geometric changes in patients with FMR. In these imaging modalities, MSCT has been reported to be valuable in studying precise mitral valvular and left ventricular (LV) geometry, including PM positions. ${ }^{8,12}$ We previously developed an anatomic image creation software system for MSCT, which provides $3 \mathrm{D}$ visualization of the mitral apparatus including subvalvular geometry. ${ }^{13}$ In addition, this system can measure mitral tenting volume, which has been thought to be the strongest determinant of MR severity. Accordingly, the purpose of the present study was to investigate whether IPMD can affect mitral valvular tenting and MR severity independently of PM tethering distance using MSCT in patients with LVD by using of our anatomic image creation software system.

\section{METHODS}

\section{Patient Population}

We retrospectively analyzed 108 consecutive patients who underwent MSCT coronary angiography with retrospective gating, which allows reconstruction of the data at desired phases of the cardiac cycle and were diagnosed by echocardiography as having regional or global LVD (ejection fraction $<50 \%$ ). The indication for MSCT coronary angiography was suspected or known coronary artery disease in all patients. After we excluded patients with the following: (1) structurally abnormal mitral valve, (2) technically inadequate images to allow analysis of 3D geometry, or (3) atrial fibrillation, 83 patients were subjected to the 3D analysis; 66 patients with old myocardial infarction and 17 patients with nonischemic dilated cardiomyopathy (DCM). Of these, 37 patients ( 25 old myocardial infarction and 12 DCM patients) had significant ( $\geq$ moderate) FMR, referred to as $\mathrm{FMR}(+)$, and 46 patients (41 old myocardial infarction and 5 DCM patients) did not, referred to as FMR(-). Figure E1 represents patient selection for the study. A total of 6 patients were excluded because images were technically inadequate to allow analysis of 3D geometry.

This study was approved by the Institutional Review Board of Kobe City Medical Center General Hospital.

\section{MSCT Protocol}

All of the patients underwent scanning on the 64-slice MSCT scanner (VCT, GE Medical Systems, Wis), which is the same protocol as for

\section{Mitral Annular Area and Geometry of Mitral Apparatus}

All images were analyzed by 2 experienced readers (K.K. and S.K.). We analyzed the end-systolic data set. A 3D reconstruction was performed using a commercially available Digital Imaging and Communications in Medicine (DICOM) viewer and the image analysis was performed with our 3D computer software, which is based on MATLAB (The MathWorks, Inc, Natick, Mass). Details of the technique recently were reported. ${ }^{13}$ We created anatomic 3D images of the mitral annulus, leaflets, and PMs to measure annular geometry, leaflet tethering, tenting height, and tenting volume. Anteroposterior (AP) annular diameter, commissure-commissure annular diameter, and mitral annular area were evaluated as previously reported. ${ }^{10,13}$ The maximum tenting height was defined as a distance from the level of the annular plane to the most tethered leaflet site. The tenting volume was calculated as a volume enclosed between the annulus and mitral leaflets. The leaflet tethering distances were measured as the distances between both the PM tips and the saddle horn and the IPMD was measured as the distance between the tips of the PMs. If PM heads were equally separated, each PM head was assessed separately and the mean position was used for the analysis. In addition, 3D coordinates of these points were determined on these images using a reference system with the origin of the centroid of the annular markers, the positive anterior axis in the annular plane passing from the middle of the posterior annulus to the midanterior annulus marker, and the positive apical axis perpendicular to the annular plane. The annular plane was determined as the least-squares fitting plane to the annulus. With this $3 \mathrm{D}$ coordinate system, positions of PM tips were resolved into their apical and nonapical (posteromedial or posterolateral) components (Figure E2).

\section{Statistical Analysis}

Continuous variables are described as means \pm standard deviations. Categoric variables were compared with the chi-square test or the Fisher exact test as appropriate. Group comparison of continuous variables was performed by one-way analysis of variance with the Tukey post hoc test.

We assessed univariate regression analysis with tenting volume and MR severity as dependent variables, and we assessed mitral geometric measurements as independent variables. Then, multivariate analysis based on stepwise multiple linear regression was performed among variables with significant univariate correlations $(P<.05)$. Because an association was present between the IPMD and posterior PM tethering distance, a multiple regression analysis was used to adjust for posterior PM tethering distance. To assess whether a threshold was present, multivariate models using analysis of covariance (ANCOVA) were used to estimate the mean (standard error [SE]) VC width and tenting volume across tertiles of IPMD. 
Normal

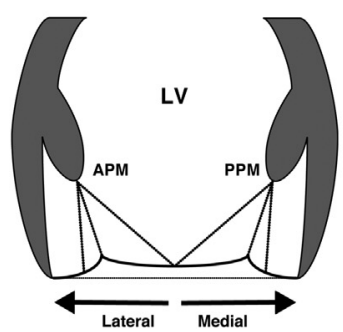

Effects of Mediolateral Shift

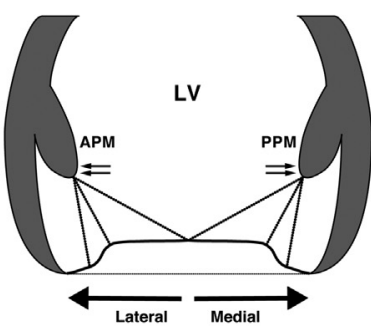

Effects of Apical Shift

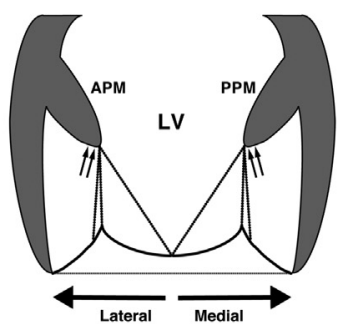

FIGURE 1. Potential mechanism of IPMD affecting mitral leaflet tethering. Left panel: A cross-sectional image of the mitral valve in a commisurecommisure direction is shown. Middle panel: Mediolateral displacement of the anterior and posterior PM, which increases the IPMD, potentially lifts up the central portion of the leaflets and increases the tethering of whole leaflets. Right panel: In contrast, apical displacement of both PMs with shortening of the IPMD does not lift up the central portion of both leaflets and results in less leaflet tethering. APM, Anterior papillary muscle; $P P M$, posterior papillary muscle; $L V$, left ventricular.

The reproducibility for the assessment was evaluated by repeated measurements by an experienced observer at 2 different time points and by a second experienced observer. Intraobserver and interobserver agreements for these measurements were evaluated by Bland-Altman analysis. Furthermore, intraclass correlation coefficients were used as indicators of reproducibility.

All statistical analyses were performed with SPSS software (version 17.0; SPSS, Inc, Chicago, Ill).

\section{RESULTS}

\section{Baseline Characteristics and Geometry of Mitral Apparatus}

Table 1 summarizes the patient characteristics and geometry of the mitral apparatus. Posterolateral and posteromedial components of both the anterior and posterior PM tethering distance were significantly larger in $\operatorname{FMR}(+)$ than in FMR(-) patients, whereas there were no significant differences in apical components of both the anterior or posterior PM tethering distance between the groups. In patients with LVD, IPMD strongly correlated with end-systolic volume index $(\mathrm{r}=0.726, P<.001)$ and end-diastolic volume index $(\mathrm{r}=0.752, P<.001)$.

Subsequently, we divided the patients on the basis of IPMD tertiles. As a result, the first (lowest) tertile consisted of IPMDs of $19.7 \mathrm{~mm}$ or less, the second (middle) tertile consisted of IPMDs greater than 19.7 and $23.4 \mathrm{~mm}$ or less, and the third (highest) tertile consisted of IPMDs greater than $23.4 \mathrm{~mm}$. Figure 2 suggests that there is a threshold for the relation of IPMD with MR severity and mitral tenting volume. ANCOVA analyses showed that patients with IPMDs in the third tertile had a significantly higher degree of MR (mean \pm SE VC widths, $4.5 \pm 0.3 \mathrm{~mm}$ ) compared with those in the first and second tertiles, adjusting for PM tethering distance (mean $\pm \mathrm{SE}$ VC widths, $3.0 \pm 0.4$ and $2.9 \pm 0.3 \mathrm{~mm}$, respectively). In addition, patients with IPMDs in the third tertile had a significantly larger tenting volume (mean \pm $\mathrm{SE}, 3.5 \pm 0.3 \mathrm{~mL}$ ) compared with those in the first and second tertiles, adjusting for PM tethering distance (mean \pm SE, 1.9 \pm 0.3 and $1.8 \pm 0.2 \mathrm{~mL}$, respectively).
Figure 3 shows anatomic and 3D images of the mitral apparatus in a FMR(+) patient with an IPMD in the third tertile and a FMR $(-)$ patient with an IPMD in the second tertile. The posterior PM tethering distance of these patients was the same length.

\section{Determinants of Mitral Tenting Volume and MR Severity}

Table 2 summarizes determinants of MR severity and mitral tenting volume in all patients with LVD. The posterior PM tethering distance and IPMDs showed a strong

TABLE 1. Patient characteristics and geometry of mitral apparatus

\begin{tabular}{|c|c|c|c|}
\hline & $\begin{array}{c}\operatorname{FMR}(-) \\
(n=46)\end{array}$ & $\begin{array}{c}\mathbf{F M R}(+) \\
(\mathbf{n}=\mathbf{3 7})\end{array}$ & $\boldsymbol{P}$ \\
\hline Age (y) & $66 \pm 11$ & $66 \pm 12$ & .891 \\
\hline Sex, male/female & $38 / 8$ & $27 / 10$ & .214 \\
\hline Prior myocardial infarction $(\%)$ & $27(59)$ & $12(32)$ & .017 \\
\hline Chronic heart failure $(\%)$ & $10(22)$ & $24(65)$ & $<.001$ \\
\hline Previous PCI (\%) & $29(63)$ & $16(43)$ & .072 \\
\hline Previous cardiac surgery $(\%)$ & $24(52)$ & $15(41)$ & .291 \\
\hline LVEDVI (mL/m²) & $62 \pm 22$ & $96 \pm 32$ & $<.001$ \\
\hline $\operatorname{LVESVI}\left(\mathrm{mL} / \mathrm{m}^{2}\right)$ & $37 \pm 18$ & $66 \pm 28$ & $<.001$ \\
\hline Ejection fraction $(\%)$ & $42 \pm 8$ & $33 \pm 10$ & $<.001$ \\
\hline MR severity (vena contracta width, $\mathrm{mm}$ ) & $1.9 \pm 0.8$ & $5.1 \pm 1.3$ & $<.001$ \\
\hline Annular AP diameter (mm) & $25 \pm 3$ & $29 \pm 5$ & $<.001$ \\
\hline Annular CC diameter (mm) & $25 \pm 3$ & $29 \pm 5$ & $<.001$ \\
\hline Mitral annular area $\left(\mathrm{cm}^{2}\right)$ & $8.7 \pm 1.4$ & $10.4 \pm 2.4$ & $<.001$ \\
\hline Anterior PM tethering distance (mm) & $29 \pm 5$ & $31 \pm 5$ & .083 \\
\hline Apical components & $23 \pm 4$ & $24 \pm 5$ & .536 \\
\hline Posterolateral components & $17 \pm 5$ & $19 \pm 4$ & .022 \\
\hline Posterior PM tethering distance $(\mathrm{mm})$ & $33 \pm 5$ & $37 \pm 5$ & $<.001$ \\
\hline Apical components & $25 \pm 4$ & $27 \pm 4$ & .118 \\
\hline Posteromedial components & $20 \pm 5$ & $25 \pm 5$ & $<.001$ \\
\hline IPMD, mm & $20 \pm 5$ & $26 \pm 7$ & $<.001$ \\
\hline Tenting volume $(\mathrm{mL})$ & $1.5 \pm 0.7$ & $3.4 \pm 1.8$ & $<.001$ \\
\hline
\end{tabular}

$P C I$, Percutaneous coronary intervention; LVEDVI, left ventricular end-diastolic volume index; LVESVI, left ventricular end-systolic volume index; $A P$, anteroposterior; $C C$, commissure-commissure; $M R$, mitral regurgitation; $I P M D$, interpapillary muscle distance; $P M$, papillary muscle; $F M R$, functional mitral regurgitation. 

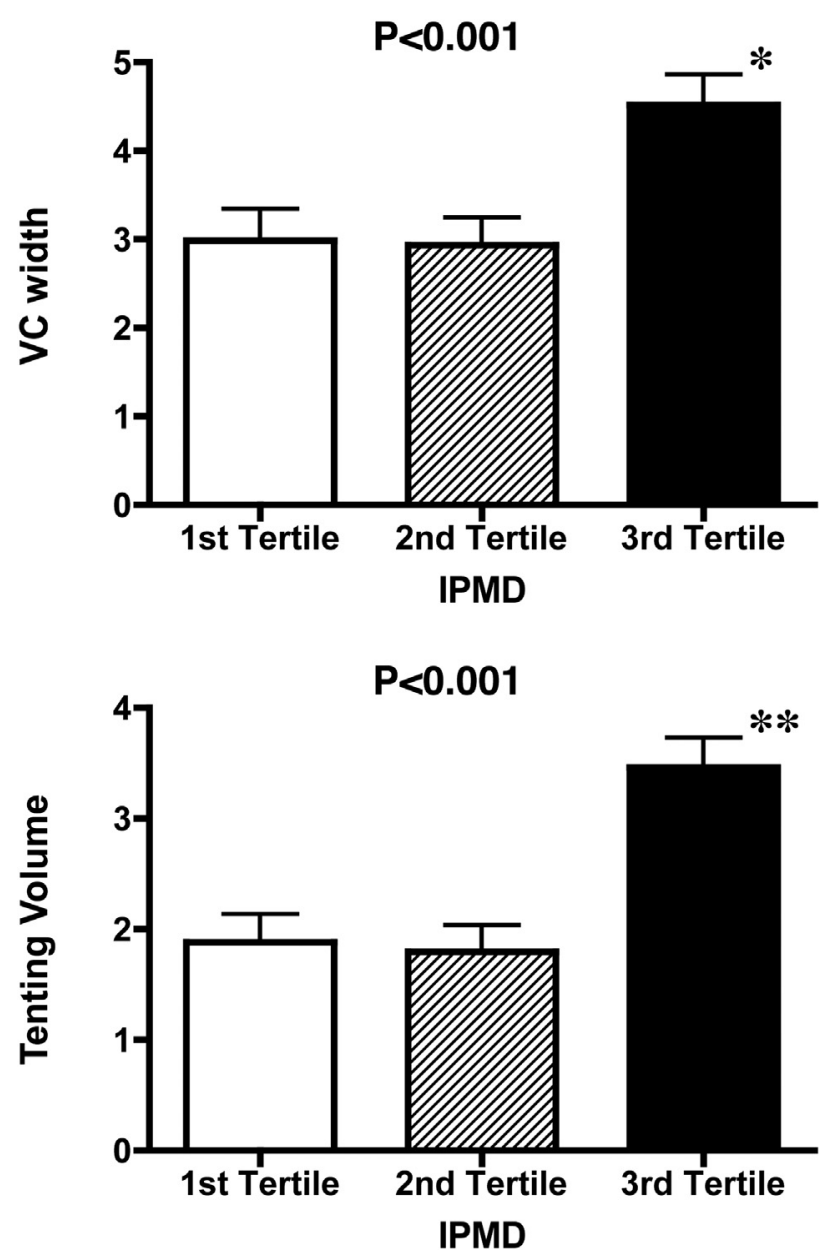

FIGURE 2. Severity of FMR and mitral tenting volume in tertiles of IPMD. Upper panel, MR severity (vena contracta width, $\mathrm{mm}$ ) in tertiles of IPMD, adjusting for posterior PM tethering distance. Lower panel, Mitral tenting volume in tertiles of IPMD, adjusting for posterior PM tethering distance. ${ }^{*} P=.005$ versus the first tertile and $P=.001$ versus the second tertile. $* * P<.001$ versus the first and the second tertiles. $V C$, Vena contracta; IPMD, interpapillary muscle distance.

correlation with MR severity. Multivariate analysis showed AP diameter and IPMD distance were the strongest determinants of both MR severity and mitral tenting volume.

\section{Reproducibility Data}

In 15 randomly selected patients, reproducibility data were assessed. The intraobserver agreement for AP and commissure-commissure diameter, lateral PM and medial PM tethering distance, and tenting volume was acceptable. The average differences were $4.7 \% \pm 3.1 \%, 4.1 \% \pm$ $4.0 \%, 4.2 \% \pm 3.3 \%, 2.3 \% \pm 2.0 \%$, and $7.2 \% \pm 6.3 \%$, respectively, and the intraclass correlation coefficients for each comparison were 0.96, 0.97, 0.95, 0.95, and 0.99, respectively. Similarly, agreement of the measurements by 2 different observers was acceptable. The average differences were $4.6 \% \pm 3.2 \%, 6.5 \% \pm 5.2 \%, 6.1 \% \pm$ $3.4 \%, 3.6 \% \pm 2.5 \%$, and $12.1 \% \pm 5.4 \%$, respectively, and the intraclass correlation coefficients for each comparison were $0.96,0.92,0.92,0.92$, and 0.98 , respectively.

\section{DISCUSSION}

In the present study, 3D visualization of the entire mitral apparatus with the use of MSCT showed that IPMD, which affects the severity of FMR independently of PM tethering distance, was the major determinant of both mitral tenting volume and MR severity. Our system provides detailed mitral geometry including annular dilatation, leaflet tethering, and PM displacement, which potentially can guide surgical therapies aimed at reducing FMR.

It has been reported that leaflet tethering by the displaced PMs owing to regional or global LV dysfunction has been suggested as the main mechanism of FMR. ${ }^{3}$ In particular, the posterior PM tethering distance has been suggested as a major determinant of MR severity. ${ }^{2-4}$ Therefore, several surgical techniques relocating the posterior PM close to the mitral annulus, which shortens the posterior PM tethering distance, have been developed to relieve leaflet tethering. ${ }^{18-22}$ On the other hand, several clinical studies have shown the PM imbrication or approximation technique was effective for reducing FMR..$^{5-8}$ Shudo et al ${ }^{8}$ showed that changes of IPMD were correlated strongly with changes of tenting height in patients who underwent PM imbrication. However, the 3D geometric relationship between IPMD and leaflet tethering, which determines MR severity, has not been well investigated. In addition, because this technique necessarily shortens posterior PM tethering distance to a certain degree by relocating the posterior PM in the inward direction, whether shortening of the IPMD affects MR severity independently of posterior PM tethering distance has been uncertain. In the present study, ANCOVA analysis showed that IPMD affected the severity of FMR independently of the PM tethering distance. Therefore, surgical planning to correct subvalvular geometry should focus on not only displaced unilateral PM positions, but also contralateral PM positions and the distance between both PMs. Interestingly, it seems that there was a threshold in the relationship between IPMD and MR severity or mitral tenting volume. These findings suggest that increasing the IPMD might be compensated to some extent by a fixed length of tethered chordae and leaflet adaptation.

Whether leaflet tethering in an apical direction can affect MR severity is still debated. In the clinical setting, a 2D echocardiographic study showed that apical displacement of PMs, as well as posterior displacement, lead to excess valvular tenting, which is associated most strongly with FMR. ${ }^{1}$ On the other hand, previous animal studies have suggested that the posterior shift of posterior PMs, rather 
FMR(+)
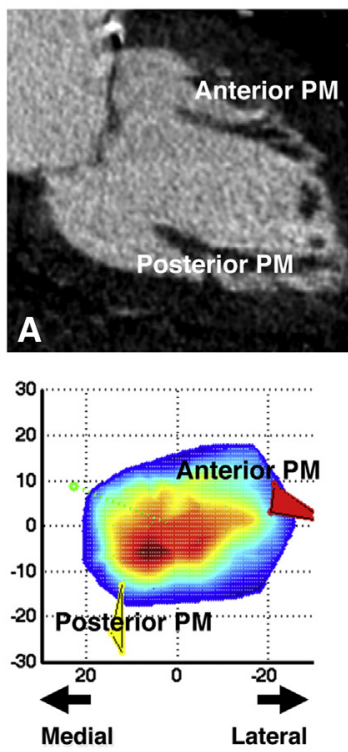

B

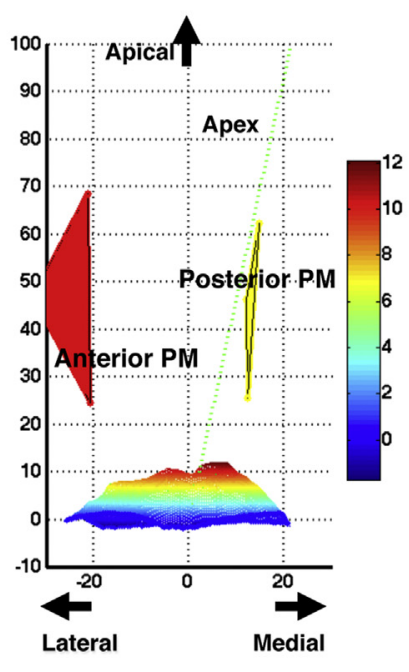

C
FMR(-)
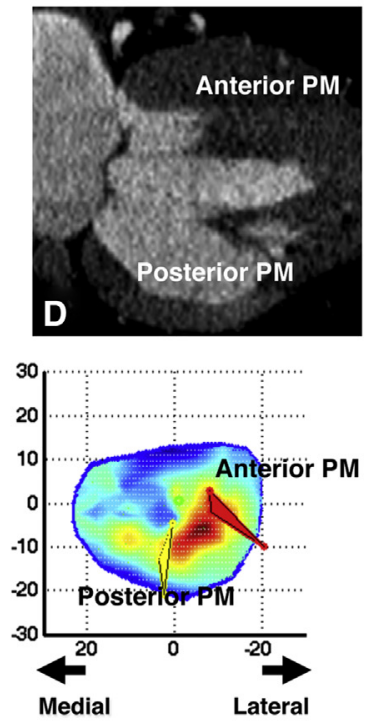

E

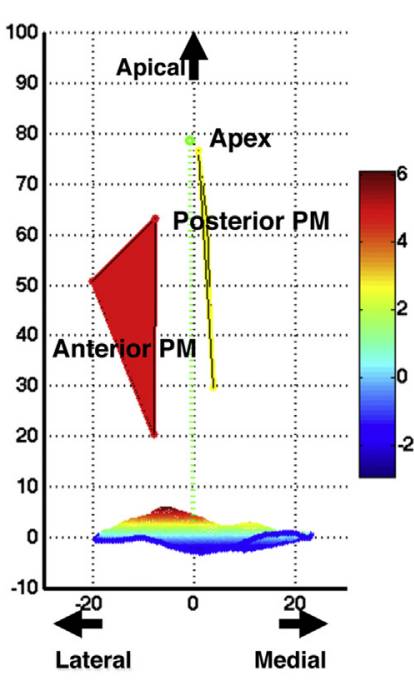

$\mathbf{F}$

FIGURE 3. Anatomic and 3D images of leaflet tethering in patients (A-C) with FMR and (D-F) without FMR. A and D, Double oblique anatomic image passing through mitral leaflets and both PMs in a mediolateral direction. B and E, Vertical view of reconstructed 3D images of mitral apparatus from the left ventricle. $\mathrm{C}$ and F, Horizontal view from the anterior direction. In the FMR $(+)$ patient, the posterior PM tethering distance, IPMD, and total tenting volume were $41 \mathrm{~mm}, 36 \mathrm{~mm}$, and $5.3 \mathrm{~mL}$, respectively. In the FMR(-) patient, the posterior PM tethering distance, IPMD, and total tenting volume were $41 \mathrm{~mm}$, 24 $\mathrm{mm}$, and $1.8 \mathrm{~mL}$, respectively. Color bar in the image indicates tenting height, which is the same in each view. The closest point to the mitral annulus of each triangle indicates the tip of the PM. FMR, Functional mitral regurgitation; $P M$, papillary muscle.

than apical or medial shifts, was a dominant factor in the development of chronic ischemic MR or FMR. ${ }^{23,24}$ Their results are consistent with our study. The present study showed that posterolateral or posteromedial components of the PM tethering distance were well correlated with MR severity and mitral tenting volume, whereas the apical component showed no significant correlations. Thus, our results suggest that relocating the PM in an apical direction might be less effective in correcting FMR than relocating the PM in an inward direction, which approximates both PMs. Further investigations are necessary to clarify key elements in an optimal PM relocation procedure by comparing the outcome of surgery with the obtained subvalvular remodeling.

In a clinical setting, 2D echocardiography has been the preferred method for mitral valve analysis, and several investigators have quantified leaflet geometry and posterior PM displacement in patients with chronic ischemic MR. ${ }^{1,4,25}$ During recent years, real-time 3D transthoracic and transesophageal echocardiography have been introduced for the analysis of mitral annular and leaflet geometries. ${ }^{9,26,27}$ Because of the complex geometry of the mitral apparatus, 3D echocardiography is suited for the assessment of FMR. However, a complete 3D echocardiography examination may require multiple acquisitions from the different several transducer positions owing to an inadequate acoustic window and not all views may be obtained optimally in all patients. ${ }^{28}$ On the other hand, MSCT has been considered to be of value for assessment of mitral valve anatomy. ${ }^{8,29,30}$ An important advantage of CT compared with echocardiography is better visualization of adjacent anatomic structures including papillary muscles. MSCT enables a detailed assessment of the anatomic variations and geometry of the PMs. Besides, as shown herein, a MSCT performed for the clinical purpose of coronary artery imaging provides additional information about mitral geometry. Our novel anatomic creation software system enabled us to visualize mitral leaflet tethering in association with PM displacement. This system also provides accurate 3D measurements of mitral annular geometry, tenting volume of mitral leaflets, and PM tethering distances. These features may be helpful for understanding the pathogenesis of FMR in patients with LVD.

\section{Study Limitations}

Our study had several limitations. First, the severity of MR was assessed only by vena contracta width in the present study, which might underestimate the MR volume. However, we also showed that IPMD was the major determinant of the mitral tenting volume, which is 
TABLE 2. Three-dimensional determinants of MR severity and tenting volume in patients with LVD $(n=83)$

\begin{tabular}{|c|c|c|c|c|}
\hline & \multicolumn{2}{|c|}{ Univariate } & \multicolumn{2}{|c|}{ Multivariate } \\
\hline & $\mathbf{r}$ & $\boldsymbol{P}$ & $\beta$ & $\boldsymbol{P}$ \\
\hline \multicolumn{5}{|l|}{ Determinants of MR severity } \\
\hline Annular AP diameter (mm) & 0.608 & $<.001$ & 0.457 & $<.001^{*}$ \\
\hline Annular CC diameter (mm) & 0.508 & $<.001$ & & \\
\hline IPMD & 0.540 & $<.001$ & 0.334 & $.001 *$ \\
\hline Anterior PM tethering distance & 0.255 & .03 & & \\
\hline Posterolateral component & 0.368 & $<.001$ & & \\
\hline Apical component & 0.143 & .220 & & \\
\hline Posterior PM tethering distance & 0.492 & $<.001$ & & \\
\hline Posteromedial component & 0.563 & $<.001$ & & \\
\hline Apical component & 0.065 & .577 & & \\
\hline \multicolumn{5}{|l|}{ Determinants of mitral tenting volume } \\
\hline Annular AP diameter (mm) & 0.679 & $<.001$ & 0.496 & $<.001 \dagger$ \\
\hline Annular CC diameter (mm) & 0.590 & $<.001$ & & \\
\hline IPMD & 0.632 & $<.001$ & 0.412 & $<.001 \dagger$ \\
\hline Anterior PM tethering distance & 0.337 & .002 & & \\
\hline Posterolateral component & 0.359 & .001 & & \\
\hline Apical component & 0.189 & .087 & & \\
\hline Posterior PM tethering distance & 0.500 & $<.001$ & & \\
\hline Posteromedial component & 0.535 & $<.001$ & & \\
\hline Apical component & 0.204 & .065 & & \\
\hline
\end{tabular}

$\beta$, Standardized regression coefficient; $C C$, commissure-commissure; $M R$, mitral regurgitation; $A P$, anteroposterior; $I P M D$, interpapillary muscle distance; $P M$, papillary muscle. $* \mathrm{R}^{2}$ of the model selected for multivariable analysis, $0.459 . \dagger \mathrm{R}^{2}$ of the model selected for multivariable analysis, 0.597 .

correlated strongly with MR severity. Thus, we believe that IPMD was the major determinant of MR severity as well as mitral tenting volume. Second, because we retrospectively analyzed patients who underwent MSCT for suspected coronary artery disease, there may be a possible selection bias. Besides, we did not investigate whether our technique is useful for the guidance of surgical procedures. Further prospective studies with MSCT are needed. Third, because the present study was retrospective, we could not validate MSCT measurements with other imaging modalities such as 3D echocardiography. However, we found a good reproducibility of MSCT measures displayed by a low interobserver and intraobserver variability. Thus, we believe that isotropic high resolution of the $3 \mathrm{D}$ matrix obtained with MSCT allows a precise assessment of mitral geometry. Finally, we did not analyze the associations between MSCT measurements and clinical outcome. Further prospective studies are needed to determine the prognostic values of MSCT measurements.

\section{References}

1. Yiu SF, Enriquez-Sarano M, Tribouilloy C, Seward JB, Tajik AJ. Determinants of the degree of functional mitral regurgitation in patients with systolic left ventricular dysfunction: a quantitative clinical study. Circulation. 2000;102: 1400-6.

2. Otsuji Y, Handschumacher MD, Liel-Cohen N, Tanabe H, Jiang L, Schwammenthal E, et al. Mechanism of ischemic mitral regurgitation with segmental left ventricular dysfunction: three-dimensional echocardiographic studies in models of acute and chronic progressive regurgitation. $J$ Am Coll Cardiol. 2001;37:641-8.

3. Otsuji Y, Handschumacher MD, Schwammenthal E, Jiang L, Song JK, Guerrero JL, et al. Insights from three-dimensional echocardiography into the mechanism of functional mitral regurgitation: direct in vivo demonstration of altered leaflet tethering geometry. Circulation. 1997;96:1999-2008.

4. Kumanohoso T, Otsuji Y, Yoshifuku S, Matsukida K, Koriyama C, Kisanuki A, et al. Mechanism of higher incidence of ischemic mitral regurgitation in patients with inferior myocardial infarction: quantitative analysis of left ventricular and mitral valve geometry in 103 patients with prior myocardial infarction. J Thorac Cardiovasc Surg. 2003;125:135-43

5. Menicanti L, Di Donato M, Frigiola A, Buckberg G, Santambrogio C, Ranucci M, et al. Ischemic mitral regurgitation: intraventricular papillary muscle imbrication without mitral ring during left ventricular restoration. J Thorac Cardiovasc Surg. 2002;123:1041-50.

6. Hvass U, Tapia M, Baron F, Pouzet B, Shafy A. Papillary muscle sling: a new functional approach to mitral repair in patients with ischemic left ventricular dysfunction and functional mitral regurgitation. Ann Thorac Surg. 2003;75: 809-11.

7. Matsui Y, Suto Y, Shimura S, Fukada Y, Naito Y, Yasuda K, et al. Impact of papillary muscles approximation on the adequacy of mitral coaptation in functional mitral regurgitation due to dilated cardiomyopathy. Ann Thorac Cardiovasc Surg. 2005;11:164-71.

8. Shudo Y, Matsumiya G, Sakaguchi T, Miyagawa S, Yoshikawa Y, Yamauchi T, et al. Assessment of changes in mitral valve configuration with multidetector computed tomography: impact of papillary muscle imbrication and ring annuloplasty. Circulation. 2010;122:S29-36.

9. Watanabe N, Ogasawara Y, Yamaura Y, Kawamoto T, Toyota E, Akasaka T, et al. Quantitation of mitral valve tenting in ischemic mitral regurgitation by transthoracic real-time three-dimensional echocardiography. J Am Coll Cardiol. 2005;45:763-9.

10. Kaji S, Nasu M, Yamamuro A, Tanabe K, Nagai K, Tani T, et al. Annular geometry in patients with chronic ischemic mitral regurgitation: three-dimensional magnetic resonance imaging study. Circulation. 2005;112:I409-14.

11. Jensen H, Jensen MO, Smerup MH, Vind-Kezunovic S, Ringgaard S, Andersen NT, et al. Impact of papillary muscle relocation as adjunct procedure to mitral ring annuloplasty in functional ischemic mitral regurgitation. Circulation. 2009:120:S92-8.

12. Delgado V, Tops LF, Schuijf JD, de Roos A, Brugada J, Schalij MJ, et al Assessment of mitral valve anatomy and geometry with multislice computed tomography. JACC Cardiovasc Imaging. 2009;2:556-65.

13. Kim K, Kaji S, An Y, Yoshitani H, Takeuchi M, Levine RA, et al. Mechanism of asymmetric leaflet tethering in ischemic mitral regurgitation: $3 \mathrm{~d}$ analysis with multislice CT. JACC Cardiovasc Imaging. 2012;5:230-2.

14. Kwan J, Shiota T, Agler DA, Popovic ZB, Qin JX, Gillinov MA, et al. Geometric differences of the mitral apparatus between ischemic and dilated cardiomyopathy with significant mitral regurgitation: real-time three-dimensional echocardiography study. Circulation. 2003;107:1135-40.

15. Zoghbi WA, Enriquez-Sarano M, Foster E, Grayburn PA, Kraft CD, Levine RA, et al. Recommendations for evaluation of the severity of native valvular regurgitation with two-dimensional and doppler echocardiography. J Am Soc Echocardiogr. 2003;16:777-802.

16. Hall SA, Brickner ME, Willett DL, Irani WN, Afridi I, Grayburn PA. Assessment of mitral regurgitation severity by Doppler color flow mapping of the vena contracta. Circulation. 1997;95:636-42.

17. Hung J, Papakostas L, Tahta SA, Hardy BG, Bollen BA, Duran CM, et al Mechanism of recurrent ischemic mitral regurgitation after annuloplasty: continued LV remodeling as a moving target. Circulation. 2004;110: II85-90.

18. Kron IL, Green GR, Cope JT. Surgical relocation of the posterior papillary muscle in chronic ischemic mitral regurgitation. Ann Thorac Surg. 2002;74: 600-1.

19. Langer F, Schafers HJ. Ring plus string: papillary muscle repositioning as an adjunctive repair technique for ischemic mitral regurgitation. $J$ Thorac Cardiovasc Surg. 2007;133:247-9.

20. Masuyama S, Marui A, Shimamoto T, Nonaka M, Tsukiji M, Watanabe N, et al Chordal translocation for ischemic mitral regurgitation may ameliorate tethering of the posterior and anterior mitral leaflets. J Thorac Cardiovasc Surg. 2008;136: 868-75.

21. Hung J, Solis J, Guerrero JL, Braithwaite GJ, Muratoglu OK, Chaput M, et al A novel approach for reducing ischemic mitral regurgitation by injection of a 
polymer to reverse remodel and reposition displaced papillary muscles. Circulation. 2008;118:S263-9.

22. Langer F, Kunihara T, Hell K, Schramm R, Schmidt KI, Aicher D, et al. Ring+string: successful repair technique for ischemic mitral regurgitation with severe leaflet tethering. Circulation. 2009;120:S85-91.

23. Tibayan FA, Rodriguez F, Zasio MK, Bailey L, Liang D, Daughters GT, et al. Geometric distortions of the mitral valvular-ventricular complex in chronic ischemic mitral regurgitation. Circulation. 2003;108:II116-21.

24. Jensen H, Jensen MO, Smerup MH, Ringgaard S, Sorensen TS, Andersen NT, et al. Three-dimensional assessment of papillary muscle displacement in a porcine model of ischemic mitral regurgitation. $J$ Thorac Cardiovasc Surg. 2010;140:1312-8.

25. Kubota K, Otsuji Y, Ueno T, Koriyama C, Levine RA, Sakata R, et al. Functional mitral stenosis after surgical annuloplasty for ischemic mitral regurgitation: importance of subvalvular tethering in the mechanism and dynamic deterioration during exertion. J Thorac Cardiovasc Surg. 2010;140:617-23.
26. Chaput M, Handschumacher MD, Tournoux F, Hua L, Guerrero JL, Vlahakes GJ, et al. Mitral leaflet adaptation to ventricular remodeling: Occurrence and adequacy in patients with functional mitral regurgitation. Circulation. 2008; $118: 845-52$.

27. Saito K, Okura H, Watanabe N, Obase K, Tamada T, Koyama T, et al. Influence of chronic tethering of the mitral valve on mitral leaflet size and coaptation in functional mitral regurgitation. JACC Cardiovasc Imaging. 2012;5:337-45.

28. Lang RM, Badano LP, Tsang W, Adams DH, Agricola E, Buck T, et al. EAE/ASE recommendations for image acquisition and display using three-dimensional echocardiography. J Am Soc Echocardiogr. 2012;25:3-46.

29. Messika-Zeitoun D, Serfaty JM, Laissy JP, Berhili M, Brochet E, Iung B, et al. Assessment of the mitral valve area in patients with mitral stenosis by multislice computed tomography. J Am Coll Cardiol. 2006;48:411-3.

30. Alkadhi H, Bettex D, Wildermuth S, Baumert B, Plass A, Grunenfelder J, et al. Dynamic cine imaging of the mitral valve with 16-mdct: A feasibility study. AJR Am J Roentgenol. 2005;185:636-46. 


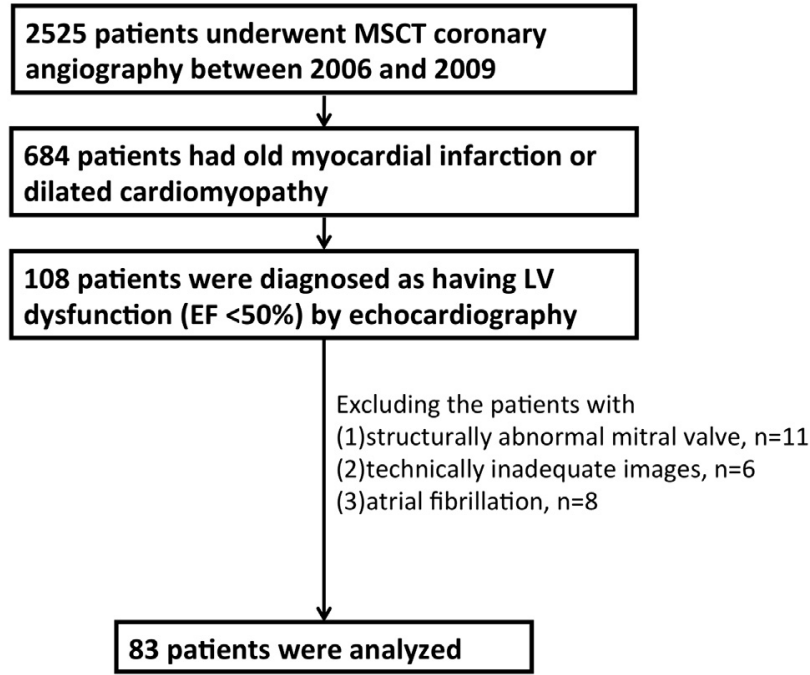

FIGURE E1. Flow chart of patient selection. During the study period, 684 patients with old myocardial infarction or dilated cardiomyopathy underwent MSCT coronary angiography. Of these, 108 patients were diagnosed as having regional or global LVD (EF $<50 \%$ ) by echocardiography. After excluding patients as shown in the figure, 83 patients were enrolled in this study. MSCT, Multislice computed tomography; $L V$, left ventricular.

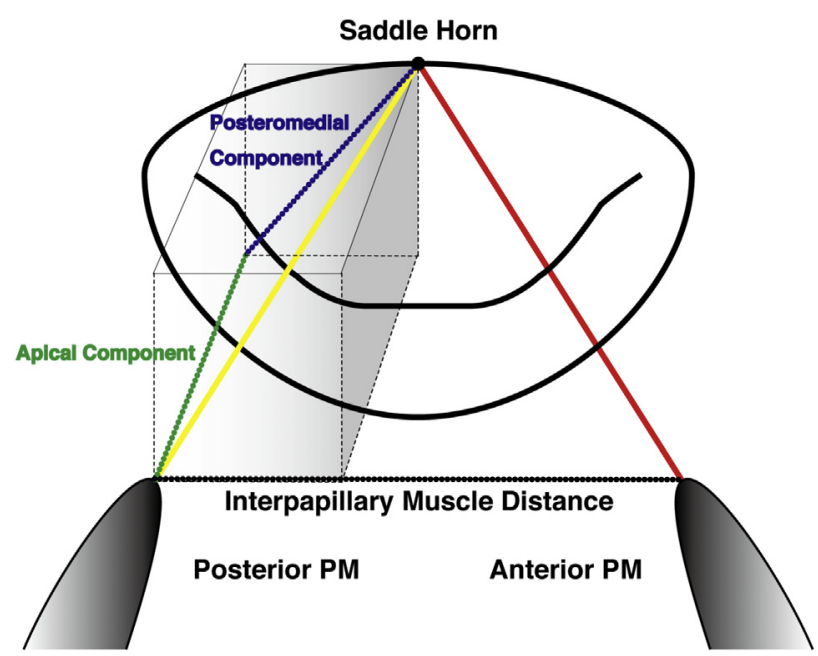

FIGURE E2. Schematic representation of anterior and posterior PM tethering distance and IPMD. Posterior PM tethering distances were measured as the distances between the posterior PM tip and the saddle horn (yellow line), and anterior PM tethering distances were measured as the distances between the anterior PM tip and the saddle horn (red line). IPMD was measured as the distance between the tips of the PMs (black dotted line). The posterior PM tethering distances were resolved into apical (green dotted line) and posteromedial (blue dotted line) components. Similarly, anterior PM tethering distances were resolved into apical and posterolateral components. PM, Papillary muscle. 